Original Article

\title{
A Study of Missile Injuries Abdomen in Shree Birendra Hospital, Chhauni
}

Hamal BK* Kansakar R*

\section{Abstract}

Treatment of missile injuries as a result of ongoing combat scenario in the country has been a great challenge. We have analyzed a total 1752 cases of trauma casualties admitted in Birendra hospital, Chhauni since November 2001. Majority of cases comprised of polytrauma. Of the 1239 patients admitted in the department of surgery $172(10 \%)$ had major injuries of which $59(31 \%)$ had abdominal missile injuries that underwent laparotomy. The predominant age group was 20 40 yrs $(80 \%)$ and most them were males. The causes being blast injuries ( $44 \%$ and bullet injuries $(56 \%)$. The most common sites of injury were in the small bowel $(26.9 \%)$ and large bowel (23.4\%) followed by injuries to the liver $(8.6 \%)$, diaphragm $(7.8 \%)$ and spleen (5.2\%). Around 26 cases (44\%) were unstable at the time of presentation. The common post operative complications were wound infection, anastomotic leak and fistula formation. There were 6 mortalities $(10 \%)$. The most common associated injuries to the chest and limbs. From $2^{\text {nt }}$ World war to the Vietnam war mortality from penetrating gunshot wounds decreased from $42-9 \%$.

Eariy evacuation, proper triage, initial resuscitation followed by definitive surgical treatment by an experienced team is beneficial thus preventing complications and mortality.

(Keywords: gunshot wound, GSW, missile injury, intra-abdominal injuries.)

At Dr. B.K Hamai, MBBS, MS

Col. Consultant Surgeon

Shree Bircndra Hospital, Chhauni

* De R. Kansakar

Surgical Resident, NAMS

\section{Introduction}

As technology advanced the incessant desire of humans for land, riches and power has spurred the development of increasingly lethal weaponry. This quest culminated with the weapons of mass destruction threatening the world today. In a global setting, abdominal missile injuries results principally from military actions and wars so has it started in Nepal since the past few years. During the Somme campaign alone of world. War I, a staggering 40,000 casualties accumulated in a 24 hour period; more than half the casualties had penetrating abdominal injuries. As a weapons advanced so has the management of patients with penetrating abdominal trauma. In Nepal however, missile injuries have been on the rise just in the past few years due to the Maoist insurgency. The surgeons have to deal with a new entity which was very much uncommon 5-10 years back. As the maximum numbers of casualties from the combat are evacuated and brought to the Shree Birendra Hospital it has been dealing with these injuries with the utmost expertise the country.

\section{Materials and Methods}

A retrospective study of 59 cases was conducted to analyze the missile injuries abdomen from November 2001 to December 2004 a period of 3 years. All the cases apart from missile injuries to the abdomen that underwent laparotomy were excluded. A through study of the injury pattern, location of injury, condition at the time of presentation, operative management, complications and mortality was done. 


\section{Results}

The total number of casualties treated in the hospital according to our hospital database since November 2001 was 1752 . Among which 1350 (77\%) were serving soldiers, 106 from the armed police force and 34 from the civil police 190 civilians and 3 foreign nationals.

Among the total number of casualties 1239 were admitted in the department of surgery and rest of them were in the department of orthopaedic surgery. Among them 1067 (86\%) had minor injuries and underwent simple debridement and dressing. 172 of them had major injuries that had to undergo major surgical procedure under general or spinal anaesthesia. Among the major injuries abdominal, head and spinal injuries were the most common being $59(31 \%)$ and $52(30 \%)$ respectively followed by soft tissue, thoracic and vascular injuries.
Among 59 cases that sustained abdominal. most of them were in the age group of 20-40 $(80 \%), 4$ in the age group of $40-60$ years.

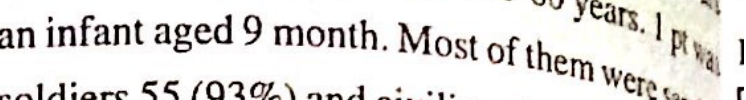
soldiers $55(93 \%)$ and civilians $4(7 \%)$. the female ratio was 55:4.

The patients that sustained gunshot wounds Among the total casualties $26(45 \%)$ were unsistbly at the time of presentation. $48(81 \%)$ of the pation were Air/Heli evacuated.

Most of the cases were that of polytrauma with the hallmark of modern warfare injury. Among the abdominal injuries most of them had multio origa involvement with polytrauma, which takes mis injuries to a greater height and challenge to to treating surgeon. The organs involved that the surgical treatment modalities are depicted in th table below.

\begin{tabular}{|l|r|l|}
\hline \multicolumn{1}{|c|}{ Organ involved } & No. of cases & \multicolumn{1}{c|}{ Operative Procedure } \\
\hline Small bowel & $31(26.9 \%)$ & $\begin{array}{l}\text { Repair-18, Resection anast-11, } \\
\text { Gastro Jejunostomy-1, Hemicolectomy-1 }\end{array}$ \\
\hline Large Bowel & $27(23.4 \%)$ & $\begin{array}{l}\text { Colostomy-12, Primary Repair-11, } \\
\text { Colo-Colic anast -1, Hemicolectomy-1 }\end{array}$ \\
\hline Liver & $10(8.6 \%)$ & Repair-9, Perithepatic packing-1 \\
\hline Diaphragm & $9(7.8 \%)$ & Repair-9 \\
\hline Retropritoneal Haematoma & $7(6 \%)$ & Conservative-6, Drainage-1 \\
\hline Spleen & $6(5.2 \%)$ & Splenectomy-6 \\
\hline Mesentery & $6(5.2 \%)$ & Conservative-6 \\
\hline Stomach & $5(4.3 \%)$ & Repair-5 \\
\hline Urinary Bladder+Ureter & $4(3.4 \%)$ & Repair-4 \\
\hline Vessel injury & $4(3.4 \%)$ & Ligation-4 \\
\hline Kidney & $3(2.6 \%)$ & Nephrectomy-2,Conservative-1 \\
\hline Gall bladder & $2(1.7 \%)$ & Cholecystectomy-2 \\
\hline Uterus & $1(0.6 \%)$ & Hysterectomy-1 \\
\hline
\end{tabular}


Multiorgan Involvement (44\%)

Injuries associated with large bowel

\begin{tabular}{|l|r|}
\hline Small bowel & 9 \\
\hline Small Bowel,Stomach,Diaphragm & 1 \\
\hline Small Bowel, Liver,Bladder & 1 \\
\hline Small Bowel, Kidney & 1 \\
\hline $\begin{array}{l}\text { Duedenum,Stomach } \\
\text { Liver, Gall Bladder, Diaphragm }\end{array}$ & 1 \\
\hline Liver, Spleen, Kidney & 1 \\
\hline Spleen,Diaphragm & 1 \\
\hline Spleen & 1 \\
\hline Bladder & 1 \\
\hline Stomach, Liver & 1 \\
\hline
\end{tabular}

Other multiorgan injuries

\begin{tabular}{|l|r|}
\hline Liver, Diaphragm & 1 \\
\hline Small Bowels, Bladder, Uterus, Illiac vein & 1 \\
\hline Spleen, Diaphragm & 2 \\
\hline Small Bowel, Ureter & 1 \\
\hline Small Bowel, Diaphragm & 1 \\
\hline Liver, Gall Bladder & 1 \\
\hline Stomach, Diaphragm & 1 \\
\hline
\end{tabular}

Polytrauma $34(58 \%)$

\begin{tabular}{|l|r|}
\hline Chest with Haemo/Pneumothorax & 15 \\
\hline Fracture Peripheral Bones & 15 \\
\hline Soft Tissue Injury & 7 \\
\hline Fracture Pelvis/Sacrum & 3 \\
\hline Spinal injury & 2 \\
\hline Eye & 2 \\
\hline Head & 1 \\
\hline
\end{tabular}

Complications (18\%)

\begin{tabular}{|l|r|}
\hline Cutaneous Fistula & 2 \\
\hline Faecal Fistula & 3 \\
\hline Wound Infection with GI Bleeding & 1 \\
\hline Wound Infection & 4 \\
\hline Anastomatic Leak & 1 \\
\hline
\end{tabular}

Relaparatomy $\quad 4(6.7 \%)$

Mortality $\quad 6(10 \%)$

\section{Discussion}

Pathophysiology: A gunshot wound is caused by a missile propelled by combustion of powder. It implies high-energy transfer and unpredictablility of the extent of intra-abdominal injuries. Not only is the missile track unpredictable, but also, secondary missiles such as bone fragments or fragments of the bullet are capable of inflicting additional injuries. The missile velocity of military firearms is much higher than that of civilian handguns and therefore has a much higher energy transfer. Missiles are categorized by their velocity profile into low-, medium-, and high-velocity projectiles. The impact speed is affected by several factors, including target distance, missile velocity, missile mass, missile shape, and drag. A missile creates longitudinal and horizontal shock and shear waves as it traverses different media. These, in turn, lead to cavitations of surrounding structures. The temporary cavity formed can contuse and lacerate tissues, fracture vascular intimae, and rupture large vascular conduits. Dependent upon the energy imparted by the missile, these injuries may extend a significant distance from the bullet track.

\section{Pre-hospital Care}

One of the main goal of pre-hospital care is rapid transport to the closest appropriate facility following evaluation of the ABCs. This concept is known as "scoop and run" most of our patients are heli/air lifted and some are transported to the regional hospitals. Still early evacuation of the casualties is very difficult due to lack of enough transport facilities and difficult terrain. Pre-hospital personnel (mainly the health workers and doctors in the field ambulance) need to be well trained in properly assessing patients and transporting them to the closest appropriate facility or trauma center. Our hospital is notified so as to alert the appropriate staff and be prepared to receive the patient.

\section{Emergency Department Care}

* Resuscitation of trauma patients with penetrating abdominal injury is based on advanced trauma life support (ATLS) protocols.

$\therefore$ Insertion of large-bore catheters into the upper extremities is done and investigations sent, or if necessary a central venous access is obtained. Lower extremity venous access in not recommended. 
* Initial fluid management is done with crystalloids. Fluid preferably is warmed to avoid hypothermia. Fluids can be infused rapidly using pressure bags.

* Blood replacement is done with typespecific blood available immediately in the ED is good practice.

* Initial resuscitation of patients with penetrating abdominal injuries depends on the condition of the patient upon arrival.

* A Secondary survey is incomplete without log-rolling the patient to each side and inspecting the back, buttocks, posterior legs, scalp, posterior neck, and perineum along with a rectal and vaginal examination. Failure to roll the patient to each side may result in a missed injury.

* A placement of a Foley catheter to monitor urine output and to check for haematuria also as the patients may have discomfort during the examination, along with a nasogastric tube (NGT) to evaluate for intra-gastric blood and to decompress the stomach.

* Efforts are started to limit hypothermia as soon as the patient arrives with prewarmed fluids, and blankets to cover the patient.

\section{* Broad spectrum intravenous antibiotics are given.}

History of the patient gives us clues to the likely injury like the associated weapon, range, position of the patient at the time of injury, which helps describe the trajectory and path of the injuring object. Also assessment of secondary injuries is vital, as may victims sustain a blunt assault or fall from various heights after sustaining a missile injury. Transport time, Initial vitals, Initial resuscitation, Last meal, medications received and Co morbid medical conditions information all help in further management.

\section{Investigations and Diagnostic Techniques}

Immediate hemoglobin, blood group and cross match Is sent but acute bleeding frequently does not reflect the hemoglobin level until fluid resuscitation is in progress. Around 3 to 6 units of blood is arranged for immediate replacement in patents who arrive in a state of profound shock.
The imaging needs of each patient differ, depend $i_{f_{17}}$ on hemodynamic stability and associated injuries

* Chest and abdominal X-rays are done $f_{0}$ all patients with penetrating abdominal trauma to ascertain presence of thoracic injury, gas under diaphragm, to seek retained foreign bodies, fractures etc.

* CT scan of the abdomen is done in stable patient's wounds to the flank and back as it provides information about solid organs and retroperitoneal structures and also violation of the peritoneum, and free fluid in the abdomen.

* Patients though rarely used in our setup now undergo abdominal ultrasound to detect free fluid as a focused abdominal sonography for trauma (FAST) it merely identifies the fluid's presence. Four views are used in trauma, with visualization of the right upper quadrant, left upper quadrant, pelvis, and pericardium. Ultrasound is up to $95 \%$ sensitive for helping detect intra-abdominal hemorrhage however it has limited role in penetrating trauma.

* DPL or local wound exploration laparoscopy can be used as diagnostic modalities for unstable patients in whom intra-abdominal injury is suggested.

Diagnostic evaluation should never delay laparotomy in any patient with a clear indication for operation, such as suspected intra-abdominal hemorrhage or peritonitis. Regardless of the mode of injury, absolute indications for laparatomy is due to hemodynamic instability, major vascular injury. evisceration, peritoneal signs, pneumoperitoneum. evidence of diaphragmatic injury, significani intraperitoneal blood (e.g. DPL findings). Approximately $85 \%$ of abdominal wall GSWs penetrates the abdominal cavity, and $95 \%$ require a surgical procedure for correction.

\section{Surgical therapy}

The most commonly injured organs associated with penetrating injuries are the small intestine $(29 \%)$. liver $(28 \%)$, and colon (23\%). A generous midline incision is given with thorough inspection of eact organ. 
Small bowel: This accounts for the maximum number beings 31 cases $26.9 \%$ of the total injuries. Examination of the entire small bowel for signs of injury is necessary. Most wounds were repaired primarily, resection Anastomosis was done in those cases which had large tears or with multiple holes in a short segment. One of our patients had 40 holes in the small bowel alone.

Duodenum: Duodenal injuries are one of the most dreaded. Exposure of the retroperitoneal part with Kochers maneuver is important and in large injuries diverticularization with drainage is important.

Colon: Among the 27 cases with colonic Injury 12 colostomies and 11 primary repair were done. Primary repair was done in patients who had small perforations with no hypotension contamination was not considered as a contraindication and it has shown good results with no anestomotic leakage. Mobilization of the retroperitoneal portion of the colon is necessary for intraoperative evaluation of this organ.

Stomach: Stomach injuries accounted for $4.3 \% 5$ cases. Examination of the stomach, both the anterior and posterior wall are important most of the injuries are repaired primarily so as in our case. This should also be suspected in left lower thoracic injuries.

Liver: Among the 10 patients who sustained liver injuries 9 underwent primary repair except 1 who had extensive bleeding and had to undergo perihepatic packing and second look operation. Control of the haemorrhage remains a critical component of the successful management of liver injuries. High-velocity bullets tend to cause burst injuries with distant contusions and parenchyma disruption. Several techniques can be used to control bleeding like Pringles maneuver, intrahepatic omental packing, finger-fracture technique. Coagulopathy occurring in the immediate postoperative period are responsible for $80 \%$ of the death in patients with liver injury.

Spleen: Usually in missile injuries there is vascular injury, extensive fragmentation, total avulsion or associated severe injuries with massive bleeding which are indications for splenectomy. Among the 6 splenic injuries all of them underwent splenectomy.

Diaphragm: Diaphragmatic injuries are usually thoracoabdominal penetrating injuries which are associated with usually haemopneumothorax and liver, stomach or splenic injury. All defects should be repaired because later enlargement of the defects may cause diaphragmatic herniation. Insertion of a chest tube and laparotomy generally is sufficient whereas thoractomy may also be needed sometimes.

\section{Damage control surgery}

Ninety percent of preventable deaths in trauma patients are related to shock from inadequate recognition of intra-abdominal haemarrhage caused by solid viscus injury. Damage control involves rapid laparotomy to control major injuries, followed by temporary closure of the abdomen and another exploration after the patient is rewarmed and stabilized. With the use of technique, approximately $40 \%$ of critically injured patients can be saved. Upon entering the abdominal cavity, 4-quadrant packing with laparotomy pads is necessary. Manual compression of the sub diaphragmatic aorta may be instituted. At this moment, the operation is stopped and blood/fluid resuscitation if performed. Subsequent unpacking of the 4-quadrants helps in identification of injuries. Vascular injuries are controlled with clamping and ligation, bowel injuries are stapled across and no attempt at any primary repair is made. Liver and retroperitoneal injuries are controlled with packing alone. The abdomen is closed rapidly with either towel clips or running suture. The patients then is transported to the recovery room and actively rewarmed and resuscitated. We operate within 24-48 hours and perform definitive repair of the injured organs. This though an old concept still needs to advocated in our setup.

\section{Postoperative period}

Postoperative management depends on intraoperative findings. The most challenging patients who have undergone damage control need to stabilized before definitive surgery. Blood replacement therapy should be performed according to massive transfusion protocols, with 6 units platelets and 2 units of fresh frozen plasma given for every 5 units of packed RBCs. Patients require active rewarming and close electrolyte monitoring. Closely record the output of all drains and tubes. Patients with evidence of ongoing bleeding do not benefit from expectant management and at times, may be candidates for angiopraphic embolization. 
General ward care of these patients focuses on wound care, fluid balance, nutrition, pain control, physical therapy, muscular reconditioning needs.

\section{Complications}

Complications are fairly frequent in this high-risk patient population and are associated with the presence of hypotentation, polytrauma, combained solid and hallo viscus inury, major vascular injury, reoperation, multiple co morbid diseases, prehospital near exsanguinations, massive blood component transfusion. Clearly, all of these factors related to impaired tissue perfusion, wound healing capacity and immune surveillance. With preoperative antibiotics therapy, the incidence of post operative infection is $8 \%$ for hollow viscus perforations and 5\% without GI tract injury. Peritoneal space may be heavily contaminated; the fascia usually is closed, while the skin remains open to undergo delayed primary closure or healing by secondary intention. Our complication tare is quite high $18 \%$ espcutially. due to refusal or delayed closure of wounds and inadequate postoperative facilities.

Early complications induced coagulopathy, ongoing bleeding and abdominal compartment syndrome. Intermediate complications induce sepsis, acute respiratory distress syndrome, pneumonia, intraabdominal collection, wound infection and enterocutaneous fistula. Late complications induce small bowel obstruction and incisional hernias.

\section{Mortality}

The death rate from penetrating trauma spans the entire spectrum (0-100\%), depending on the extent of injury. Victims incur an expected mortality rate of approximately $25 \%$, which clearly influenced by perhospital hypotention, exsanguination and arrest in the field or on presentation. Death generally occur within the first 72 hours from hypo perfusion and its sequelae. Death also may occur two or more weeks later from complications related to sepsis, the systemic inflammatory response syndrome (SIRS) or multiple organ dysfunction syndrome. We have a relatively low mortality rate $(10 \%)$ partly because the very serious patients do not make it on time whereas most mortality is in patients with polytrauma with multiple organ failure.

\section{Missed injuries}

Missed injuries occur most commonly in minimally or maximally injured patients the foemer due to minimal physical findings and the later due 10 diversion of attention of life-threatening priorities Penetrating trauma to the abdomen that requires operative therapy typically has small missed-injury rate. The patients who tend or have missed injuries are those with flank or back wounds that are judged superficial or diaphragmatic, duodenal and retroperitoneal injuries.

\section{Conclusion}

Management of abdominal missile injury is basically a teamwork starting from early evacuation and assessment by the perhospital medical personnel to the ER management, operative procedure, post operative care and rahabilitation by an experienced trauma team. With a systemic multimodality approach and better cooperation improve our trauma care system and thus decrease our mortality to some extent.

\section{References}

1. Peizam AB, Schwab CW, Rhodes M: The Trauma Manual $2^{\text {nd }}$ ed. Williams \& Wilkins; 2002:236-295.

2. Trunkey DD, Lewis FR: Current Therapy of Trauma $2^{\text {ndd }}$ ed. B.C. Decker, 1986:262. 309.

3. Komar AR: abdominal trauma, Penetrating update 2004 Nov Emedicine.

4. Morris JA Jr, Eddy VA, Rutherford EJ:The trauma celiotomy: the evolving concepts of damage control. Curr Probl Surg 1996 Aug; 33(8): 611-700 Medline.

5. Kaplan LJ: Abdominal Penetrating. Emedicine

6. Cayten CG, Nassoura ZE: Abdomen In: Ivatury $R R$, ed. The Text Book of Penetrating Trauma. $1^{\text {st }} \mathrm{ed}$. Willams \& Wilkins; 1996:281-299.

7. Uppot RN: Bowel Trauma 2001 Dec Emedicine

8. Welsford M Diaphragmatic Injuries 2004 Nov. Emedicine

9. Azer SA Intestinal Perforation 2003 Mar Emedicine

10. Khan AN: Liver Trauma 2004 Juni Medicine

11. Sahani R: Penetrating Chest Trauma 200Nov. Emedicine 